\title{
Prototipo de App de seguimiento escolar de Estudiantes de Ingenieria en Sistemas Computacionales del Tecnm Campus Oaxaca
}

\section{School Monitoring App Prototype for Computer Systems Engineering Students of the Tecnm Campus Oaxaca}

\author{
ALTAMIRANO-CABRERA, Marisol†*, BENITEZ-QUECHA, Claribel, DIAZ-LARA, Carlos Alberto \\ y DIAZ-SARMIENTO, Bibiana
}

Tecnológico Nacional de México Campus Oaxaca y Tecnológico Nacional de México Campus Iguala

ID $1^{\mathrm{er}}$ Autor: Marisol, Altamirano-Cabrera / ORC ID: 0000-0001-5800-9655, CVU CONACYT ID: 657390

ID 1 ${ }^{\text {er }}$ Coautor: Claribel, Benitez-Quecha / ORC ID: 0000-0001-6516-5760, CVU CONACYT ID: 657582

ID $2^{\text {do }}$ Coautor: Carlos Alberto, Diaz-Lara / ORC ID: 0000-0002-1782-1900

ID $3^{\text {er }}$ Coautor: Bibiana, Diaz-Sarmiento / ORC ID: 0000-0003-4350-6311, CVU CONACYT ID: 820776

DOI: $10.35429 / J T A E .2020 .12 .4 .23 .31$

Recibido: 30 de Junio, 2020; Aceptado 30 de Octubre, 2020

Resumen

El Proyecto plantea una solución a los problemas académicos que se presentan en los departamentos estudiantiles y en la rendición de cuentas de los directivos del plantel, por no alcanzar los indicadores recomendados del porcentaje de egreso o la disminución de los porcentajes de deserción escolar que se manifiestan por el bajo desempeño en estudiantes. Desarrollada para el seguimiento académico de los alumnos de la Ingeniería en Sistemas Computacionales (ISC) del Tecnológico Nacional de México (TecNM) campus Oaxaca para dismunir la problemática. Este prototipo sustituye algunos procesos que se llevan a cado de forma manual, lo que se traduce en reducción de gastos y de tiempo, sumado al hecho de que el estudiante tambien tendrá acceso de manera rápida y detallada a su situación académica y las sugerencias en otras áreas (tutorias). La solución planteada "Sistema de Alertas Tempranas (SAT) para estudiantes de la ISC del TecNM Campus Oaxaca mediante una web apps informará sobre el status académico de los estudiantes basados en los lineamientos de acreditación de materias emitidos por el Tecnológico Nacional de México. La metodología utilizada para el análisis y diseño del sistema fue la investigación de forma aplicada, la recolección de información mediante cuestionarios y entrevistas. Al final de la investigación se obtuvo aplicación web y movil para el apoyo al Departamento de Servicios Escolares, División de Estudios profesionales y Desarrollo Académico así como para el Coordinador Institucional del programa de Tutorías; en el cual se empleó a SCRUM como metodología de desarrollo ágil para el desarrollo de software. Se considera segun las pruebas inciales que el trabajo realizado aporta a la linea de investigación sobre gestión tecnología educativa, ya que la propuesta optimiza la gestión de recursos y tiempo.

Alertas temprana, Intervención Educativa, Retención

\begin{abstract}
The Project proposes a solution to the academic problems that arise in the student departments and in the accountability of the directors of the school, for failing to achieve the recommended indicators of the percentage of school leavers or the decrease in the percentages of school degree that are presented by the low performance in students with levels of conditionality (Regular student, Irregular student and Deserter); through a computer solution that provides the academic follow-up of students of Computer Systems Engineering (ISC) of the Tecnológico Nacional de México (TecNM) campus Oaxaca and support in addressing the problem. This prototype integrade some processes that are carried out manually, resulting in cost and time savings, added to the fact that the student will also have quick and detailed access to their academic situation and suggestions in other areas such as tutorial support (scholarships, counseling, psychological support) if required. The solution proposed "Early Warning System (SAT) for students of the ISC of the TecNM Campus Oaxaca through a web apps is based theoretically on research on standard processes to low performance identification, the classification according to the academic status that the accreditation guidelines of subjects issued by the National Technological of Mexico and the academic follow-up of students. The methodology used for the analysis and design of the system was applied research, the collection of information through questionnaires and interviews. At the end of the research, a web app for the academic follow-up of students was obtained to support the School Services Department, Division of Professional Studies and Academic Development as the Institutional Coordinator of the Tutoring Program; in which SCRUM was used as an agile development methodology for software development. It is considered according to the initial evidence that the work carried out contributes to the line of research on educational technology management, since the proposal optimizes the management of resources and time.
\end{abstract}

Early Alerts, Educational Intervention, Retention

Citación: ALTAMIRANO-CABRERA, Marisol, BENITEZ-QUECHA, Claribel, DIAZ-LARA, Carlos Alberto y DIAZSARMIENTO, Bibiana. Prototipo de App de seguimiento escolar de Estudiantes de Ingenieria en Sistemas Computacionales del Tecnm Campus Oaxaca. Revista de Tecnología y Educación. 2020. 4-12: 23.31

\footnotetext{
* Correspondencia del Autor (Correo electrónico: marisol_altamirano@itoaxaca.edu.mx)

$\dagger$ Investigador contribuyendo como primer autor.
} 


\section{Planteamiento del Problema}

La deserción, el atraso estudiantil, los bajos índices de desempeño y de culminación de programas de educación superior son algunas de las preocupaciones más apremiantes de instancias gubernamentales, instituciones de educación superior, personal directivo universitario, padres de familia, docentes y cuerpos académicos. Ya que con la presencia de estos problemas, se produce un fenómeno constante de repitencia por el alto índice de inasistencias y el bajo nivel de aprendizaje de los contenidos programáticos que se acumulan a lo largo de la trayectoria escolar de los estudiantes, que lo conduce finalmente a desertar y no concluir sus estudios.

Desde esta perspectiva, la retención constituye un desafío para cualquier institución de educación superior de tipo público o privado. Esta preocupación tambien se vé reflejada en los estadisticos de eficiencia terminal, indice de deserción y reprobación que el Tecnológico Nacional de México campus Oaxaca envía de forma trimestral a las oficinas centrales del TecNM.

El Sistema Integral de Información (SII, 2018) operable por el departamento de servicios escolares del Instituto Tecnológico de Oaxaca(ITO) perteneciente al Tecnológico Nacional de México (TecNM), no incluye información de los estudiantes que por alguna razón solicitan la baja (temporal o definitiva) ni sus causas; tampoco un procedimiento establecido para el seguimiento de la trayectoria académica que alerte a los directivos de la institución y actúen de manera inmediata con los estudiantes en situación de curso especial y lograr su permanencia con los conocimientos, habilidades y actitudes que se espera desarrollen.

El lineamiento indica que un estudiante debe concluir el plan de estudios, en un mínimo de siete y máximo de doce semestres; proceso que se corrompe cuando se presenta el fenómeno de repitencia y curso especial. Este fenómeno se manifiesta principalmente en las materias de programación y en las de ciencias básicas generando en el alumno una situación de estrés y desventaja escolar debido a sus dificultades académicas.
El índice de reprobación en el Instituto Tecnológico de Oaxaca, constituye un problema que cada vez va en aumento, que se visualiza desde el primer semestre y se agudiza a partir del tercero. Esta crisis hace que los jóvenes no terminen de forma regular sus estudios $\mathrm{o}$ definitivamente no completen su desarrollo y preparación académica independientemente de los programas a nivel gubernamental que ayudan a disminuirla. Por un lado la falta de interés, compromiso y disposición de los docentes en la formación académica de los estudiantes debido a la carga académica asignada en cada periodo semestral y la falta de apoyo para el trabajo tutorial por parte de los directivos; por lo que al no propiciarse el mejoramiento de la calidad educativa, no se mejoran los índices de permanencia y reprobación.; con lo anterior no es posible generar propuestas de cambio a los planes y programas de estudios y tampoco fortalecer el aprendizaje de los estudiantes para alcanzar mayores niveles de logro.

Ante la problemática descrita anteriormente y al no contar en la actualidad con información real sobre el fenómeno que impiden a los estudiantes la culminación de sus estudios en la institución, surge la necesidad de incorporar herramientas $y$ estrategias que aporten soluciones a la situación antes planteada mediante un proceso investigativo que permita conocerlos como punto de referencia y así plantear una estrategia de retención de estudiantes que estén en riesgo de abandonar la institución o que su única disyuntiva sea abandonar sus estudios y tomar deciciones acertadas referente a las necesidades detectadas.

\section{Objetivos}

\section{General}

Diseñar una propuesta sistematizada de alerta temprana para estudiantes en riesgo de abandono de la Ingeniería en Sistemas Computacionales del Tecnológico nacional de México Campus Oaxaca empleando tecnología WEB

\section{Específicos}

- Identificar el perfil académico de los estudiantes de la Ingeniería en Sistemas Computacionales con desempeño irregular. 
- Clasificar el perfil académico de los estudiantes de la Ingeniería en Sistemas Computacionales en situación de curso especial (posibles desertores).

- Identificar dificultades en el proceso de enseñanza-aprendizaje (profesores $y$ estudiantes)

- Interpretar datos obtenidos, que permitan hacer la propuesta del diseño de la alerta temprana de la Ingeniería en Sistemas Computacionales del Instituto Tecnológico de Oaxaca.

- Desarrollar un prototipo funcional a través de tecnologías web 2.0, guiado por la metodología elegida.

- Diseñar una WEB APPS en el que se detecte y dé seguimiento a los estudiantes en situación de riesgo integrando los indicadores, herramientas y procedimientos requeridos.

- Realizar pruebas funcionales sobre el software desarrollado, verificando el cumplimiento de cada una de las fases del modelo elegido.

\section{Justificación}

A nivel internacional, la eficiencia de un sistema educativo ha sido definida por la Organización de las Naciones Unidas para la Educación, la Ciencia y la Cultura de manera sucinta: "Grado en el cual un sistema educativo consigue optimizar la relación inversión-resultado en la educación" (UNESCO, 2018, s.p.).

En México, la principal función de una institución de educación superior (IES) es la docencia y, por tanto, su eficiencia depende principalmente de la proporción de alumnos que logran egresar o titularse; estos indicadores actualmente son un reto para el Tecnológico Nacional de México, quien en su Programa Institucional de Innovación y Desarrollo (PIID) 2013-2018 indica que la eficiencia terminal de todo el sistema, alcanzan un 54.33 por ciento por lo que es importante para el TecNM indagar las causas por las que cerca de la mitad de sus alumnos no logra concluir sus estudios a tiempo y resolver los problemas de reprobación y deserción de estudiantes como línea de acción.
Estos datos se reflejan en el informe de rendición de cuentas 2018 del Instituto Tecnológico de Oaxaca (IRC_ITO,2018) cuyos datos de permanencia escolar de los estudiantes de la carrera de Ingeniería en Sistemas Computacionales, generalmente es de doce semestres para contabilizarlos dentro de las estadísticas semestrales/anuales de la eficiencia terminal del instituto y que se presume que no se alcanzan por la evidencia de solicitud de prórroga para la terminación de estudios e incrementando la deserción escolar.

Se pretende que los resultados obtenidos en este proyecto de investigación puedan ser usados por el I.T. Oaxaca para diseñar e implementar actividades y/o estrategias didácticas e innovadoras que permitan mejorar el proceso de aprendizaje; con la finalidad de lograr la adecuada integración de estos alumnos en la vida académica y social de la comunidad escolar $\mathrm{y}$ valorar los requerimientos del alumnado con mayores riesgos de deserción escolar. Otro de los beneficios que se oferta con esta investigación es la posibilidad de ahorrar costos en el pago de horas extras u honorarios a profesores y pueden ser destinadas a otras áreas para mejorar la infraestructura y mantenimiento de las instalaciones.

\section{Viabilidad de la Investigación}

En la primera etapa del proyecto se realizará el análisis de datos de tipo estadístico, ya que se requieren cuantificar el porcentaje de la problemática social, las relaciones causales y su intensidad, el diseño será longitudinal ya que la recogida de información se realizará en los estudiantes que ingresaron en el año 2019 para ver su evolución. Con el fin de hacer inferencias respecto al cambio, sus determinantes y sus consecuencias.

Se propone emplear el método de Coeficiente Alfa de Cronbach: Para evaluar la confiabilidad o la homogeneidad de las preguntas (en el instrumento a aplicar) y estar seguros que no hay sesgos en las entrevistas, cuestionarios y opiniones que se obtengan de expertos y estudiantes sobre la situación académica. El Nivel de profundidad se propone que sea "Predictivo" porque se van a estudiar comportamientos controlando intervenciones y analizando resultados en diferentes condiciones con el fin de establecer efectos predecibles que ofrecen elementos para el establecimiento de normas y controles.

ALTAMIRANO-CABRERA, Marisol, BENITEZ-QUECHA, Claribel, DIAZ-LARA, Carlos Alberto y DIAZ-SARMIENTO, Bibiana. Prototipo de App de seguimiento escolar de Estudiantes de Ingenieria en Sistemas Computacionales del Tecnm Campus Oaxaca. Revista de Tecnología y Educación. 2020 
Para establecer una metodología de desarrollo ágil se debe tomar en cuenta los factores comunes que permiten que un usuario sin ser experto, pueda emplearlo, manipularlo y consultarlo sin problema alguno

Como herramienta de desarrollo de software se propone el framework Laravel, para la gestión de datos, el gestor mySQL y javaScript, y como herramienta para el modelado de procesos del negocio se propone el uso de BPMN (Business Process Model and Notation).

Para determinar que el proyecto es viable se realiza un estudio a los gastos efectuados para su realización, contemplando únicamente recursos técnicos. Ya que el recurso administrativo y humano es de la plantilla laboral del instituto. La viabilidad del proyecto se sustenta, en que ya se cuenta con equipo de cómputo para el desarrollo del proyecto; sin embargo, se plantea la adquisición de un servidor, y lenguajes de programación de licencia gratuita para la construcción del mismo.

\section{Hipótesis}

Comprobar que un sistema de la alerta temprana anticipa situaciones de riesgo en los estudiantes de la Ingeniería en Sistemas Computacionales del Tecnológico Nacional de México Campus Oaxaca.

\section{Diseño Metodológico}

El "Prototipo de App de seguimiento escolar de Estudiantes de Ingenieria en Sistemas Computacionales del Tecnm Campus Oaxaca". Es un proyecto de Investigación Social Aplicada, (Müggenburg, Pérez 2007) La investigación será de tipo cualitativa, directamente en el Instituto Tecnológico de Oaxaca con los estudiantes de la Ingeniería en Sistemas Computacionales en donde se presenta el fenómeno a estudiar. Así como a los hechos que se observan dentro de la institución; específicamente con los estudiantes del tercer semestre, en el cual se ha detectado un porcentaje alto de deserción académica, sin saber sus causas Con instrumentos como encuestas, cuestionarios y entrevistas.
Lo anterior nos permitirá estudiar esta situación para diagnosticar las necesidades y problemas que afectan a nuestros estudiantes y con ello emitir recomendaciones para evitar que este fenómeno se siga presentado en un futuro. (Ander-Egg, E, 1994)

El objetivo será establecer una propuesta sistematizada de alerta temprana para estudiantes en riesgo de abandono de la Ingeniería en Sistemas Computacionales del Instituto Tecnológico de Oaxaca estudiando sus comportamientos para el establecimiento de normas y controles.

Población Total: Estudiantes en Activo hombres y mujeres Inscritos en el Instituto Tecnológico de Oaxaca

Muestra: Estudiantes que cursan la Ingeniería en Sistemas Computacionales Unidad de análisis: Estudiantes inscritos en los semestres Enero-Junio 2019 y Agosto Diciembre 2019.

Características de la Población: Estudiantes (hombres y mujeres) en situación de riesgo académico: con una materia reprobada hasta por 2 ocasiones y estudiantes que se encuentran en situación de curso especial cuyas edades se encuentran entre los 19-24 años de edad que cursan la Ingeniería en Sistemas Computacionales (Ver figura 1)

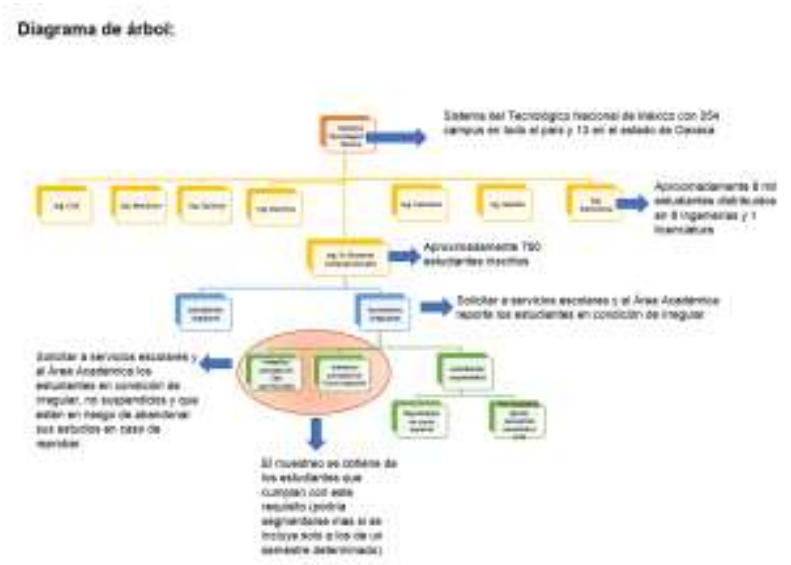

Figura 1 Diagrama de flujo de información para la obtención de la muestra

Fuente: Elaboración Propia 
El proceso incicia con la solicitud de información al departamento de servicios escolares y al área académica estudiantil en condición de irregular (materias no acreditadas, en repetición y en situación de especial) este reporte contiene el nombre completo, número de control, número telefónico y kardex académico). Con los datos capturados se calcula el tamaño de la muestra 42 estudiantes para llevar a cabo la encuesta, los cuales se contactarán para que de manera voluntaria sean partícipes de esta investigación, garantizando la confiabilidad de la información suministrada. Ya que los resultados se darán a conocer de forma integral y con ello cuidar la identificación de los entrevistados.

\section{Resultados}

Los resultados obtenidos para el cumplimento del primer objetivo de este proyecto. infiere que los estudiantes que aprobaron la implementación de un SAT en el TecNM Campus Oaxaca están entre un $58.52 \%$ y $68.48 \%$, así mismo se aprecia que el porcentaje de los Estudiantes que han reprobado al menos una materia como repetidores y generado curso especial durante su trayectoria en la institución están entre un $82.79 \%$ y $89.90 \%$.

Ambas cifras sobrepasan el $50 \%$ de la población y por ese motivo es que se puede afirmar que este proyecto es viable a nivel de alerta y seguimiento para su implementación.

Se prueba que la proporción de alumnos del TecNM campus Oaxaca que están dispuestos a aceptar la implantación de este proyecto tiene una diferencia altamente significativa con el $80 \%$ estimándose que se encuentra entre $94.79 \%$ y 98.51 .

Este proyecto tiene la finalidad de apoyar a los directivos para la mejor toma de decisiones, referente a los indicadores de deserción y reprobación escolar y que afectan directamente a los índices de egreso por cohorte de la institución.

El diseño de la interfaz se observa en las siguientes figuras

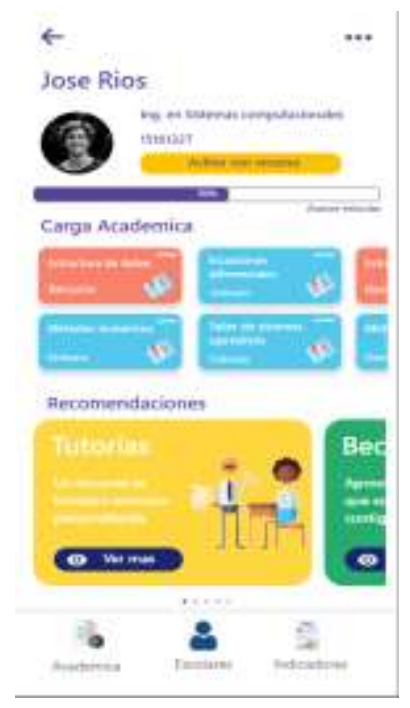

Figura 3 Interfaz2 APP-SAT (Estudiante) Fuente: Elaboración Propia

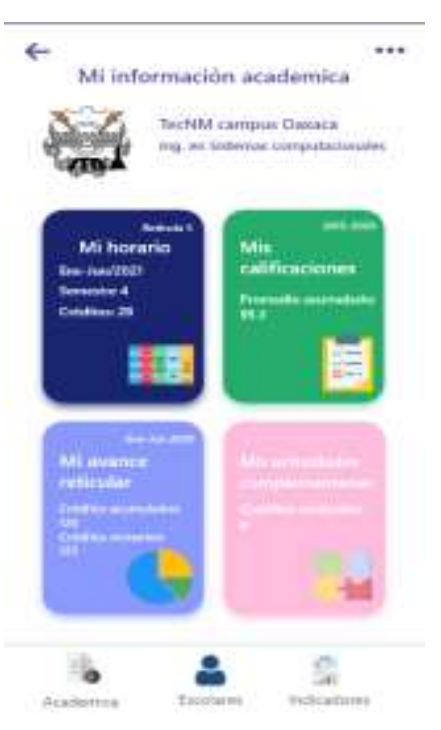

Figura 2 Interfaz1 APP-SAT (Estudiante) Fuente: Elaboración Propia

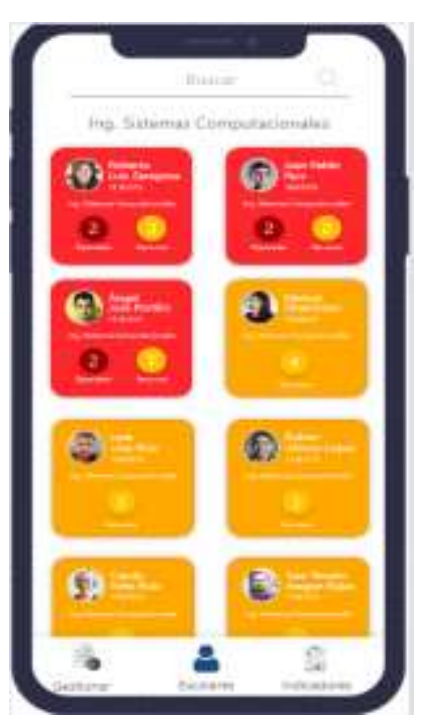

Figura 4 Interfaz3 APP-SAT (Servicios Escolares) Fuente: Elaboración Propia 


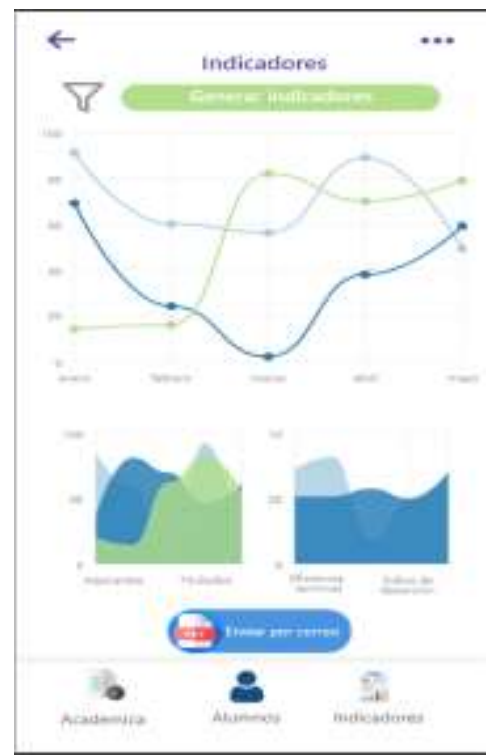

Figura 5 Interfaz3 APP-SAT (Servicios Escolares) Fuente: Elaboración Propia

\section{Conclusiones}

Al utilizar un Sistema de Alertas Tempranas el programa educativo de Ingeniería en Sistemas Computacionales del Tecnológico Nacional de México campus Oaxaca tendría la oportunidad de conocer oportunamente las condiciones académicas de los estudiantes inscritos. Además de detectar el posible riesgo de deserción de un estudiante y la posibilidad de registrar fácil y en un futuro apoyar a la coordinación institucional de tutorías del plantel para que registre ordenadamente las actividades de acompañamiento que se le realicen a los estudiantes y avanzar en el diseño de estrategias de intervención de mayor pertinencia.

Sobre el seguimiento tutorial, el $57 \%$ de los encuestados indican que la institución cuenta con un proceso de atención burocrático, como es el caso de la difusión de los programas complementarios de asesoría, por lo que la mayoría, prefiere buscar por sus propios medios en las áreas académicas correspondientes o entre sus compañeros.

La situación con respecto a la solicitud en el área de becas indica que les dan prioridad a otras situaciones y no la económica. Con rasgos y características identificados durante el desarrollo del "Prototipo de App de seguimiento escolar de Estudiantes de Ingenieria en Sistemas Computacionales del Tecnm Campus Oaxaca", se concluye que se requiere la implementación de programas o medidas preventivas $y$ remediales que contribuyan a culminar exitosamente la Ingeniería en Sistemas Computacionales.
Fortaleciendo la calidad académica de la institución, tomando en cuenta las exigencias en el ámbito nacional e internacional en este aspecto.

Finalmente Con el desarrollo de este proyecto se dio respuesta a la formulación del problema planteada inicialmente en este proyecto “¿Es eficaz una alerta temprana, para la retención de los estudiantes con riesgo de abandono en la Ingeniería en Sistemas Computacionales del Instituto Tecnológico de Oaxaca?", la respuesta es SI., una vez cumplido los objetivos propuestos.

\section{Agradecimientos}

Agradecemos al Tecnológico Nacional de México campus Instituto Tecnológico de Oaxaca e Instituto Tecnológico de Iguala, las facilidades otorgadas para la realización del proyecto. Principalmente a los estudiantes que nos proporcionaron su información y egresados que nos apoyaron con el desarrollo, su experiencia y tiempo.

\section{Referencias}

Altamirano, M. (2020).Propuesta de un sistema de alertas tempranas para estudiantes de la Ingenieria en Sistemas Computacionales del Instituto Tecnológico de Oaxaca mediante una web apps. (Tesis de Doctorado). Universidad Virtual de Estudios Superiores (UNIVES), Jalisco,México.

Amaya, A. A., Cervantes, D. C., \& Mejía, J. A. L. Capítulo 3. El nuevo perfil docente en la era digital: desafío en el contexto de la desigualdad educativa, caso estudiantes UAT. Precariedad juvenil y sus nexos con la desigualdad educativa: casos en Argentina, Colombia, México y España, 53.

Arámburo, C. A., Bañales, D. L. G., \& Cesaretti, E. H. R. Capítulo 9. Aplicación de una metodología de aprendizaje basado en proyectos para el desarrollo de sistemas web. Tecnologías de la Información en Educación: Sistematización de experiencias docentes, 103.

Avilés, G., Bascuñán, E. (2015). Estrategias para la retención estudiantil en la Educación Media en Chile. Educere, 19 (64), 737-751. 
Baena, G. (1998).Técnicas de Investigación. En instrumentos de investigación (pp. 5966).México. Editores Mexicanos Unidos

Barragán, S., González, L. （2017). Acercamiento a la deserción estudiantil desde la integración social y académica. Revista de la Educación Superior, XLVI (3) (183), 63-86.

Barranco, S.M. y Santacruz, M.C. (1995). Los egresados de la UAA: trayectoria escolar y desempeño laboral, México, Universidad Autónoma de Aguascalientes. Disponible en: https://www.worldcat.org/title/egresados-de-lauaa-trayectoria-escolar-y-desempenolaboral/oclc/40360926/editions? referer=di\&edit ions View=true

Chavarro Mendez, J. (2020). Ruta metodológica mediada por Tic para el mejoramiento de los procesos de la gestión escolar (Master's thesis, Escuela de Ingenierías).

COEPES (2018). Catálogo de la Oferta de Educación Superior en el Estado de Oaxaca 2018-2019. Comisión Estatal para la Planeación de la Educación Superior. Disponible en http://www.coepes.oaxaca.gob.mx/wpcontent/uploads/2018/07/CATALOGOLICENCIATURA-2018-2019WEB-4.pdf

Diario Oficial de la Federación (2014). Decreto que crea el Tecnológico nacional de México. DOF $2014 \quad$ Disponible en http://dof.gob.mx/nota_detalle.php?codigo $=535$ $3459 \&$ fecha $=23 / 07 / 2014 \&$ print $=$ true

Durán Urrea, Margarita María. “'Se me acabaron los créditos'. Trayectorias académicas de estudiantes de antropología en la Universidad Nacional de 92

Colombia, 1990-2012". Literatura: teoría, historia, crítica 17.2 (2015): 165-193. https://revistas.unal.edu.co/index.php/lthc/articl e/viewFile/51285/52659

Fondo de las Naciones Unidas para la Infancia (2013). Los derechos de la infancia y la adolescencia en Oaxaca. UNICEF México. Disponible en https://www.unicef.org/mexico/spanish/SITAN 2013_Oaxaca.pdf
García Robelo, Octaviano, \& Barrón Tirado, Concepción. (2011). Un estudio sobre la trayectoria escolar de los estudiantes de doctorado en Pedagogía. Perfiles educativos, 33(131), 94-113. Recuperado en 04 de julio de 2019 , de http://www.scielo.org.mx/scielo.php?script=sci _arttext\&pid=S018526982011000100007\&lng=es\&tlng=es.

Gobierno del Estado de Oaxaca (2016). Plan Estatal de desarrollo 2016-2022. Coordinación General del Comité Estatal de Planeación para el Desarrollo de Oaxaca. Disponible en https://www.finanzasoaxaca.gob.mx/pdf/planes/ Plan_Estatal_de_Desarrollo_2016-2022.pdf

Henríquez, N., Escobar, D. (2016). Construcción de un modelo de alerta temprana para la detección de estudiantes en riesgo de deserción de la universidad metropolitana de ciencias de la educación. Revista Mexicana de Investigación Educativa, 21 (71), 1221-1248.

Hernández, R., Fernández, Baptista, P. (1997). Metodología de la Investigación. Edit. McGRAW - HILL DE MÉXICO. Pág. 260-283. Hernández, J., Rojas, M., López, Y. (2016). Caracterización del desertor y diseño del sistema de alertas tempranas: Facultad de Minas, Universidad Nacional de Colombia. Revista Logos, Ciencia \& Tecnología, 8 (1), 77-87. 93

Herrera E., Araya G., Fernández H., Morera M., y Fonseca H. (2015). El diagnóstico temprano de niños y niñas con riesgo académico mediante un sistema de diagnóstico perceptual-motor: Estudio retrospectivo longitudinal de evidencias de su efectividad. Revista Electrónica Educare, 19 (3), 1-14.

Instituto Nacional para la Evaluación de la Educación (2011). Panorama Educativo de México. INEE Indicadores del Sistema Educativo Nacional.. Disponible en http://www.inee.edu.mx/bie/mapa_indica/2010/ PanoramaEducativoDeMexico/AT/AT02/2010_ AT02_d-vinculo.pdf

Instituto Nacional de Estadística y Geografía (2017).Encuesta Intercensal. Principales resultados de la Encuesta Intercensal 2017: Oaxaca $\quad \%$ Disponible en http://internet.contenidos.Inegi.org.mx/contenid os/Productos/prod_serv/contenidos/espanol/bvI negi/productos/nueva_estruc/inter_censal/estad os2015/702825079857.pdf

ALTAMIRANO-CABRERA, Marisol, BENITEZ-QUECHA, Claribel, DIAZ-LARA, Carlos Alberto y DIAZ-SARMIENTO, Bibiana. Prototipo de App de seguimiento escolar de Estudiantes de Ingenieria en Sistemas Computacionales del Tecnm Campus Oaxaca. Revista de Tecnología y Educación. 2020 
Instituto Tecnológico de Oaxaca (2017). Informe de rendición de cuentas. Disponible en http://www.itoaxaca.edu.mx/web/wpcontent/uploads/2018/03/20181_IRC2017.pdf Instituto Tecnológico de Oaxaca (2018). Disponible en

http://www.oaxaca.tecnm.mx/web/it-oaxaca/

Lineamiento para la Evaluación y Acreditación de Asignaturas versión 1.0 Planes de estudio 2009-2010. Pág. 3-4,11-12. Tecnológico Nacional de México. Disponible en https://www.tecnm.mx/docencia 94

Marín, C., Librado, E., Alarcón, M. (2015). Estudiantes universitarios en situación de examen de última oportunidad de inglés i. Revista Electrónica "Actualidades Investigativas en Educación", 15 (1), 1-20.

Müggenburg Rodríguez V., María Cristina, \& Pérez Cabrera, Iñiga (2007). Tipos de estudio en el enfoque de investigación cuantitativa. <i xmlns="http://www.w3.org/1999/xhtml" $>$ Enfer mería Universitaria, $4</ \mathrm{i}>(1)$, undefinedundefined. [fecha de Consulta 6 de Octubre de 2019]. ISSN: 1665-7063. Disponible en: <a xmlns="http://www.w3.org/1999/xhtml"

target="_blank" href=" https://www.redalyc.org/articulo.oa?id=3587/3 58741821004">

Ordoñez, M. (2000). El territorio del Estado de Oaxaca:una revisión histórica. Investigaciones geográficas, (42), 67-89. Disponible en http://www.scielo.org.mx/scielo.php?script=sci _arttext\&pid=S0188-

$46112000000200006 \& \operatorname{Ing}=e s \& n r m=i s o>$.

Ortega, J.C., López, R., y Alarcón, E. (2015). Trayectorias escolares en educación superior. Propuesta metodológica y experiencias en México. México, Universidad Veracruzana. Recuperado https://www.uv.mx/bdie/files/2016/01/LibroTrayectorias-escolares-educacion-superior.pdf

Portillo-Torres, M. (2015). Propuesta de un nuevo enfoque para reducir el abandono escolar en secundaria. Revista Electrónica Educare, 19 (2), 303-316. Disponible en http://www.redalyc.org/html/1941/1941380170 17/index.html
Rojas, A. (2017). Intervención de tres estrategias educativas para cursos de programación en educación superior. Education in the Knowledge Society, 18 (4), 21-34.

Ramos, J. (2014). Situación educativa de la población joven y adulta de América Latina y el Caribe $\mathrm{Su}$ unidad/diversidad. Revista Interamericana de Educación de Adultos, 36 (1), 10-36. 95

Rodríguez, Ariza y Ramos. (2014). Calidad institucional y rendimiento académico. El caso de las universidades del Caribe colombiano. Perfiles Educativos, XXXVI (143), 10-29.

ROMERO-FRÍAS, E. \& ALCARAZ, J.L. (2013). "Using Web 2.0 tools in accounting education. A comparison of the use of Social Networking Sites and Personal Learning Environments". 49th British Accounting and Finance Association (BAFA) Annual Conference 2013, Middlesex University London (UK), 13-15 mayo 2013.

Ruiz Cornelio,M. Palmeros,G. y Alfaro, S. (2017). Trayectorias escolares de alumnos universitarios. Un análisis a partir de los indicadores de desempeño. Disponible en: http://www.comie.org.mx/congreso/memoriaele ctronica/v14/doc/2387.pdf

Sánchez J., Urías M. y Gutiérrez B. (2015). Análisis de los problemas de aprendizaje de la programación orientada a objetos. Ra Ximhai, 11 (4), 289-304.

Secretaria de economía. (2018). Información económica y estatal (Oaxaca 2018-02). Disponible en https://www.gob.mx/cms/uploads/attachment/fi le/300346/oaxaca_2018_02.pdf

Suárez-Montes, N., \& Díaz-Subieta, L. (2015). Estrés académico, deserción y estrategias de retención de estudiantes en la educación superior. Revista de Salud Pública, 17 (2), 300$313 . \quad$ Disponible en: http://www.redalyc.org/articulo.oa?id=4224177 8013

TecNM, (2018). Programa Institucional de Innovación y Desarrollo 2013-2018. Tecnológico Nacional de México. Disponible en 96

http://www.tecnm.mx/images/areas/planeacion/ 2014/PIID_2013-2018_TECNM_Final.pdf.

ALTAMIRANO-CABRERA, Marisol, BENITEZ-QUECHA, Claribel, DIAZ-LARA, Carlos Alberto y DIAZ-SARMIENTO, Bibiana. Prototipo de App de seguimiento escolar de Estudiantes de Ingenieria en Sistemas Computacionales del Tecnm Campus Oaxaca. Revista de Tecnología y Educación. 2020 
TecNM. (2017). Sistema Integral de Información. Departamento de servicios escolares. Tecnológico nacional de México. Instituto Tecnológico de Oaxaca. Disponible en https://www.tecnm.mx/sistema-integral-deinformacion

TecNM. (2018). Ingeniería en Sistemas Computacionales. Disponible en https://www.tecnm.mx/licenciatura_2009_2010 /ingenieria-en-sistemas-computacionales

TecNM. (2018). Retícula de Ingeniería en Sistemas Computacionales. Disponible en https://www.tecnm.mx/licenciatura_2009_2010 /ingenieria-en-sistemas-computacionales.

Organización de las Naciones Unidas para la Educación, la Ciencia y la Cultura (2018). Tesauro de la UNESCO. Disponible en http://vocabularies.unesco.org/browser/thesauru s/en/page/concept6707?clang=es

Paredes, J. (2011). Deserción escolar en el Departamento de Ensenada. "Un problema que atraviesa a la sociedad Argentina actual". 9 Congreso Argentino y 4 Latinoamericano de Educación Física y Ciencias Departamento de Educación Física Facultad de Humanidades y Ciencias de la Educación Universidad Nacional de La Plata. Disponible en http://congresoeducacionfisica.fahce.unlp.edu.a r/9o-ca-y-4o-la-efyc/publicacionesactas/Paredes.-M6.pdf

Sierra Bravo, R. (1994). El diseño de la investigación y la selección del problema a investigar. Tecnicas de Investigación social. Teoría y ejercicios. Curso Proyecto de Investigación IV. Universidad Virtual [UNIVES]. pp. 1 - 9. Recuperado desde file:///C:/Users/Marisol\%20Altamirano/Deskto p/2017/Doctorado/MODULO7-SeptiembreDiciembre2019/Investigacion\%20IV/LECTUR AS/diseno.pdf 97

Universidades en Oaxaca. (2017). Disponible en https://mextudia.com/en-tuciudad/universidades-en-oaxaca/

Zárate, R., Mantilla, E. (2014). La deserción estudiantil UIS, una mirada desde la responsabilidad social universitaria. Zona Próxima, (21), 121-134. 